\title{
New Combined Technique for Fingerprint Image Enhancement
}

\author{
Alaa Ahmed Abbood ${ }^{1,4}$, Mohammed Sabbih Hamoud Al-Tamimi ${ }^{2,4}$, Sabine U. Peters ${ }^{3} \&$ Ghazali Sulong ${ }^{4}$ \\ ${ }^{1}$ University of Information Technology and communications, Baghdad, Iraq \\ ${ }^{2}$ Department of Computer Science, College of Science, University of Baghdad, Baghdad, Iraq \\ ${ }^{3}$ College of Education, Florida State University, Tallahassee, 32306 Florida, USA \\ ${ }^{4}$ UTM-IRDA Digital Media Centre (MaGIC-X), Faculty of Computing, University Technology Malaysia, 81310 \\ Skudai, Johor Bahru, Malaysia \\ Correspondence: Alaa Ahmed Abbood, University of Information Technology and communications, Baghdad, \\ Iraq. Tel: 964-772-7143-353. E-mail: alaa.ahab@hotmail.com; m_altamimi75@yahoo.com
}

Received: August 25, 2016

doi:10.5539/mas.v11n1p222
Accepted: September 6, 2016

Online Published: December 19, 2016

URL: http://dx.doi.org/10.5539/mas.v11n1p222

\begin{abstract}
This paper presents a combination of enhancement techniques for fingerprint images affected by different type of noise. These techniques were applied to improve image quality and come up with an acceptable image contrast. The proposed method included five different enhancement techniques: Normalization, Histogram Equalization, Binarization, Skeletonization and Fusion. The Normalization process standardized the pixel intensity which facilitated the processing of subsequent image enhancement stages. Subsequently, the Histogram Equalization technique increased the contrast of the images. Furthermore, the Binarization and Skeletonization techniques were implemented to differentiate between the ridge and valley structures and to obtain one pixel-wide lines. Finally, the Fusion technique was used to merge the results of the Histogram Equalization process with the Skeletonization process to obtain the new high contrast images. The proposed method was tested in different quality images from National Institute of Standard and Technology (NIST) special database 14. The experimental results are very encouraging and the current enhancement method appeared to be effective by improving different quality images.
\end{abstract}

Keywords: classification, fingerprint, identification, image enhancement, image pre-process

\section{Introduction}

The quality of the ridge and valley in a fingerprint image is an essential characteristic, as these structures contain all the information associated with fingerprint features (Yager \& Amin, 2004b). However, in real applications it is very rare to find images with perfect quality due to factors like skin variations, impression conditions and the illumination effect of capture devices. Due to these different types of noise that affect the clarity of fingerprint patterns, image enhancement techniques are often employed to reduce this noise effect and enhance the definition of ridges and valleys. Applying enhancement algorithms to fingerprint images is necessary for recovering low quality fingerprint images. For the fingerprint image quality to have good intensity there must be a high contrast between ridges and valleys. There must also be clear continuity in the ridge structures. Ideally, perfect images show alternating ridges and valleys that flow in locally constant direction. These are called high contrast images. This regularity in the fingerprint patterns facilitates the process of feature extraction. An example of a high quality fingerprint image can be seen in Figure 1(a) while low quality fingerprint images are shown in Figure 1(c). With regards to Figure 1(b), low quality images can be characterized by low contrast, and Figure 1(d) shows an over ink image with high noise levels and obvious distortions. These combined effects are known as spurious effects. 


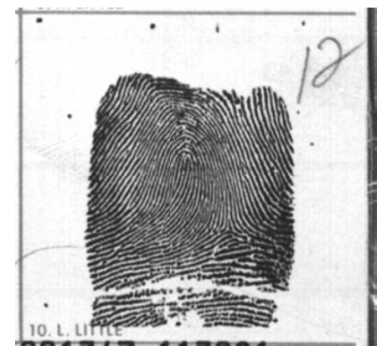

(a)

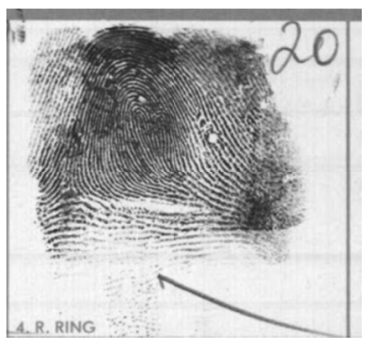

(c)

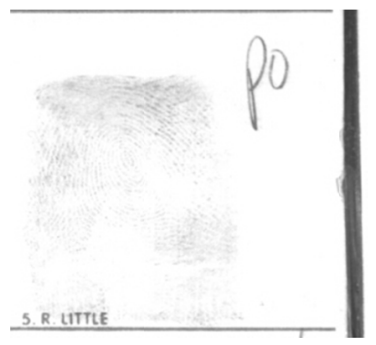

(b)

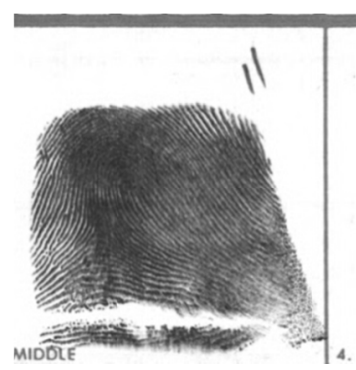

(d)

Figure 1. Quality of fingerprint images: (a) Good, (b) Low contrast (c) Wet and (d) Over ink (source NIST 14)

In order to develop a high performance Automatic Fingerprint Identification System (AFIS), image quality is a key factor (Yager \& Amin, 2004a). Fingerprint image samples are often distorted by smudges or blotches. In poor quality fingerprint images, the valleys and ridges are not clear and discontinuities exist in the ridges and are often caused by random interference in the capturing devices (A. K. Jain, Pankanti, \& Bolle, 1997). Overcoming the problems related to extreme contrast conditions (low contrast or high contrast) and associated discontinuities of the fingerprint patterns is a challenge. Therefore, it is necessary to apply fingerprint image enhancement before any processes procedure (Çavuşoğlu \& Görgünoğlu, 2008). In this paper, fingerprint images were normalized by using grayscale normalization to standardize the intensity of the pixels, and the contrasts of the images were improved using Histogram Equalization. To solve the problem of discontinuity, Binarization and Skeletonization approaches were applied. In the final step, Fusion algorithms were applied to the output image, and with the help of Skeletonization and Histogram Equalization, enhanced the quality of fingerprint images overall processes are summarized in Figure 2.

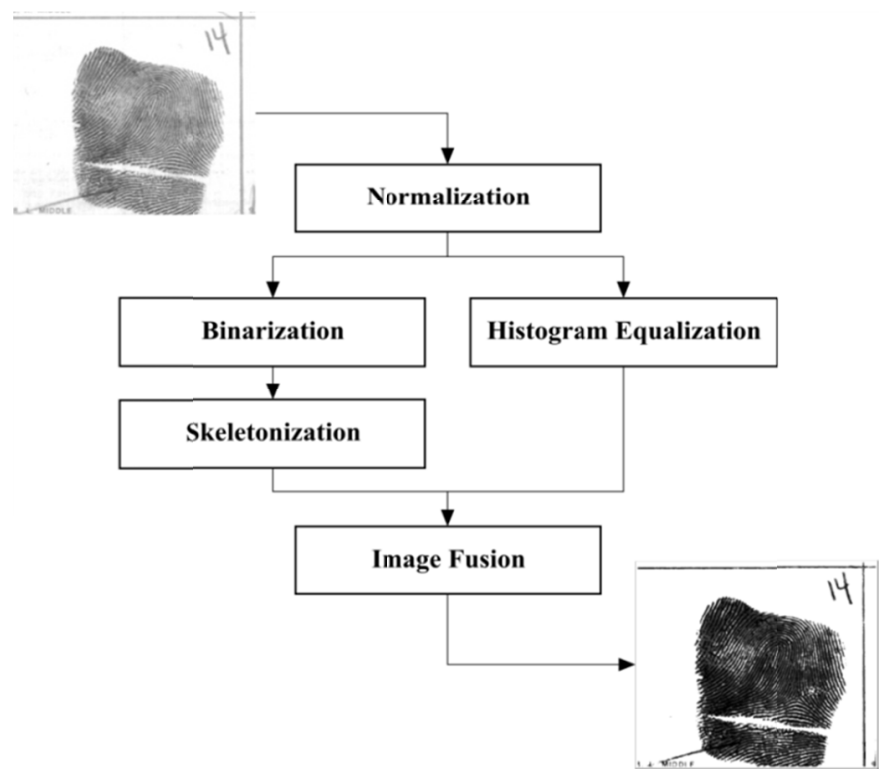

Figure 2. The proposed enhanced fingerprint image technique 


\section{Grey-level Normalization}

Pixel intensity values of fingerprint images of one part of an image may differ from that of another part due to the sensitivity of the capture device with respect to the temperature and also environmental illumination. The intensity values of the grayscale images are normally between 0 and 255. The values of the grayscale from 0 to 128 are considered lower-range, which typically results in dark images (under-exposure). The upper-range of the greyscale levels, with values from 128 to 255 , refers to the bright part of the images (over-exposure). In order to capture an image with uniform characteristics, and to remove the noise that is introduced by the sensor, as well as limiting the variation in grey-level values along the ridges and valleys, a normalization technique needs to be applied. The grayscale normalization technique is a process used to standardize the intensity level of pixels in an image by adjusting the range of grey-level values. It does so by using the statistical parameters of mean and variance. The Normalization method proposed by (A. Jain, 1998) is adopted in this study due to its efficiency and simplicity. The method consists of three steps: In the first step, a global mean value of the fingerprint image is determined. In the second step, the global variance value of the fingerprint image is computed. In the final step, new intensity values are calculated. This process detail can be summarized as follows:

i. Let $I(i, j)$ denote the grey-level or intensity value of the pixel at the $i^{\text {th }}$ row and $j^{\text {th }}$ column of $\mathrm{W} \times \mathrm{H}$ pixels of fingerprint image size.

ii. Let $M_{g}$ and $V_{g}$ denote the global mean and global variance values of $I(i, j)$, respectively as follows:

$$
\mathrm{M}_{\mathrm{g}}=\frac{1}{\mathrm{~W} \times \mathrm{H}} \sum_{\mathrm{x}=1}^{\mathrm{W}} \sum_{\mathrm{y}=1}^{\mathrm{H}} \mathrm{I}(\mathrm{x}, \mathrm{y})
$$

and

$$
\mathrm{V}_{\mathrm{g}}=\frac{1}{\mathrm{~W} \times \mathrm{H}} \sum_{\mathrm{x}=1}^{\mathrm{W}} \sum_{\mathrm{y}=1}^{\mathrm{H}}\left(\mathrm{I}(\mathrm{x}, \mathrm{y})-\mathrm{M}_{\mathrm{g}}\right)^{2}
$$

iii. Calculate the normalized grey-level value for pixel $(i, j)$ of the fingerprint image, which is denoted by $N(\mathrm{i}, \mathrm{j})$ as follows:

$$
N(x, y)= \begin{cases}M_{0}+\sqrt{\frac{V_{0}\left(I(x, y)-M_{g}\right)^{2}}{V_{g}}} & \text { if } I(x, y)>M_{g} \\ M_{0}-\sqrt{\frac{V_{0}\left(I(x, y)-M_{g}\right)^{2}}{V_{g}}} & \text { otherwise }\end{cases}
$$

Where $M_{0}$ and $V_{0}$ refer to the desired mean and variance values, respectively. Normalization is a pixel-wise operation that does not change the clarity of the ridge and valley structures. Even if normalization is performed on the entire image, this process cannot compensate for the intensity variations in different parts of the image due to finger pressure differences. For this paper the values of both $\mathrm{M}_{0}$ and $\mathrm{V}_{0}$ are set at 128 . An example of a fingerprint image that has gone through the normalization process is provided in Figure $3 a$, and $b$.

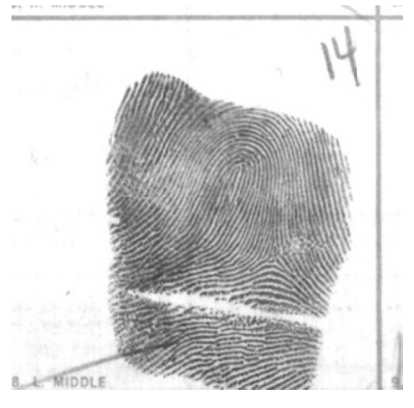

(a)

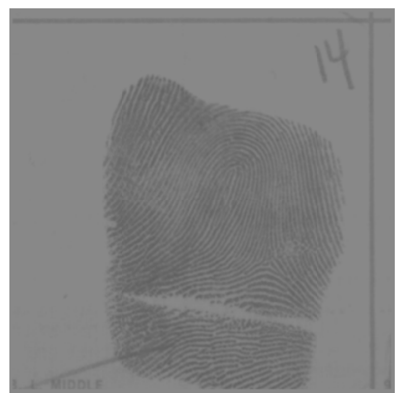

(b)

Figure 3. Grey-level normalization: (a) Original image (Source NIST Database 14: f0000008) (b) Normalized image

\section{Contrast Enhancement}

Although the Normalization process standardizes the intensity of the pixels, the problems with the image contrasts still have to be overcome. The image contrast problem can be classified as being either high contrast or 
low contrast. It is generally caused by impressions of the fingertip during an ink scan and the effect of illumination in a live scan. Therefore, a Histogram Equalization (HE) technique is applied which is considered the most common approach for improving the appearance of poor quality images. In this paper, the method that have been used was adopted from the study of (Hanoon, 2011) as follows:

$$
P_{r}\left(r_{k}\right)=\frac{n_{k}}{n}
$$

Where $\mathrm{P}_{\mathrm{r}}\left(\mathrm{r}_{\mathrm{k}}\right)$ is between 0 and $1, \mathrm{k}=0,1, \ldots, 255 \mathrm{n}_{\mathrm{k}}$ is the number of pixels at intensity level $\mathrm{r}_{\mathrm{k}}$ and $\mathrm{n}$ is the total number of pixels.

The new intensity value $S_{k}$ for level $\mathrm{k}$ is derived from the following formula:

$$
S_{k}=\sum_{j=0}^{k} \frac{n_{j}}{n}=\sum_{j=0}^{k} P_{r}\left(r_{j}\right)
$$

In other words, the values in a normalized histogram approximate the probability of occurrence of each intensity level in the image. A significant contrast differences between the normalized histogram and the equalized one illustrate the effectiveness of the HE as a principal contrast enhancement tool. The result of the Histogram Equalization Enhancement will be grayscale image as illustrated in Figure 4 (a), (b), (c) and (d).

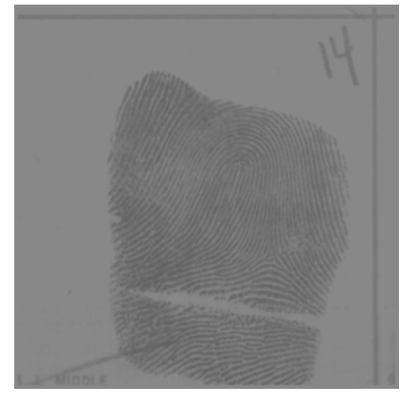

(a)

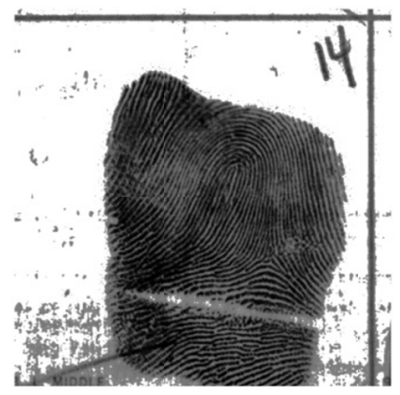

(c)

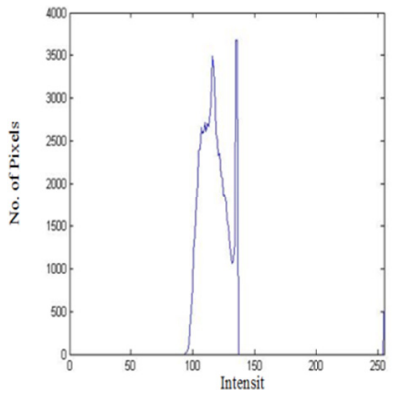

(b)

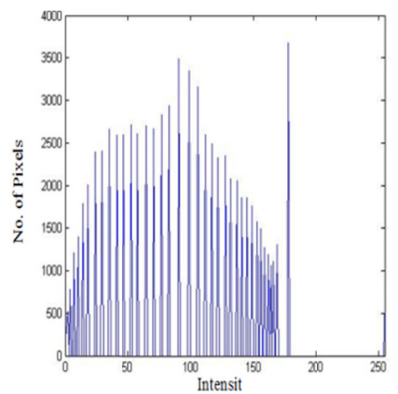

(d)

Figure 4. (a), (b) Normalized image with its histogram before HE, while (c), (d) Enhanced image after HE with its histogram

\section{Binarization}

Binarization is a process that converts the grayscale image into binary form. A pixel value of 0 is assigned to the black area of the fingerprint image that represents the ridge lines, and a pixel value of 1 identifies the white area in the images that represents the valleys. The Binarization approach is used to keep the characteristics of the fingerprint ridge structure and to remove some of the cohesion between the patterns. The final result of Binarization is a clear image. In this study, the Binarization technique used is not static, so a dynamic calculation is performed on the threshold value to fill in any existing gaps and that method was adopted from the study of (Fu, Huang, \& Xu, 2013). In other words, filling in of the gaps is accomplished by calculating the average value of the grey-level for the related pixel $P(i, j)$ in each $w \times w$ block. Then, threshold value $T=P \mp \partial$ of each block is obtained, where $\partial \in[5,10]$ (obtained from a series of experiments). The size of the block plays an 
important role in this method. In this study, a $15 \times 15$ block size was empirically chosen and used for processing of the separation. The gradient value for each pixel was compared with the threshold value. If it was larger than the threshold, a gray value of 1 was assigned, otherwise the value was 0 . Due to some of the noise in the original images which skewed the results, the fill method was applied by using a block size of $3 \times 3$ to fill in each pixel.

The process is described as follows:

1. The input fingerprint image is divided into q non-overlapping blocks of $15 \times 15$ pixels.

2. For each block, calculate the mean using Equation (3.6) as follows:

$$
\text { Ave }=\frac{1}{15 \times 15} \sum_{i}^{15} \sum_{j}^{15} I(i, j)
$$

3. Calculate the threshold $\mathrm{T}$ :

$$
T=A v e-\partial
$$

where $\partial=7$ (impirically determined)

4. Compare the middle pixel's value with $\mathrm{T}$; if the value is greater than $\mathrm{T}$, assigned a 1 , otherwise the value is 0 .

5. Repeat Step 2 until all the blocks are exhausted.

6. Upon completion of the first cycle of Binarization, following is fill-in-the-gaps procedure: a window of $3 \times 3$ with centre pixel $\mathrm{P}_{\mathrm{i}}$, denoted by Figure 5 , is used to scan the entire image from left-to-right and top-to-bottom to localize the noise (if any) using the following rules given by equations (8), (9).

\begin{tabular}{|l|l|l|}
\hline$P_{1}$ & $P_{2}$ & $P_{3}$ \\
\hline$P_{4}$ & $P_{i}$ & $P_{5}$ \\
\hline$P_{6}$ & $P_{7}$ & $P_{8}$ \\
\hline
\end{tabular}

Figurev 5. A 3x3 window: the centre pixel and its neighbours

$$
\begin{aligned}
& R_{1}=P_{1}+P_{4}+P_{6}+P_{3}+P_{5}+P_{8} \\
& R_{2}=P_{1}+P_{2}+P_{3}+P_{6}+P_{7}+P_{8}
\end{aligned}
$$

If $\left(R_{1}=0\right)$ or $\left(R_{2}=0\right)$ then $P_{i}$ is given a value of 0 which is black.

If $(R 1+R 2)>=7$ then $P_{i}$ is given a value of 1 which is white

This algorithm shows a significant improvement of the ridge structure of the fingerprint image. The dynamic threshold value was used to remove the noise in the foreground of the fingerprint image as evidently illustrated in Figure 6 (a), (b).

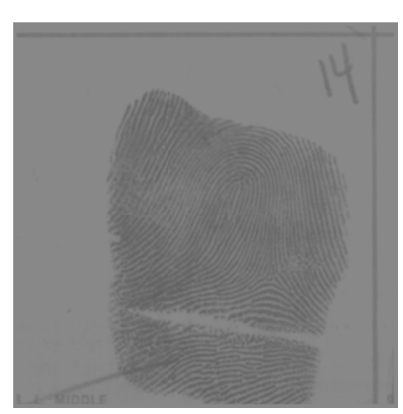

(a)

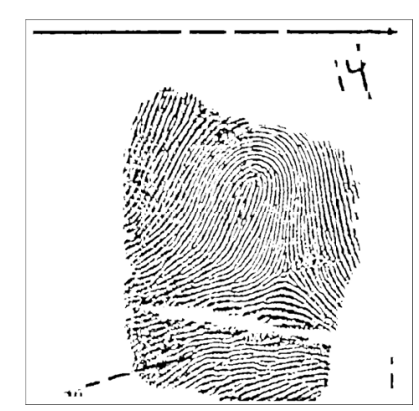

(b)

Figure 6. Binarization: (a) Normalized image, (b) Binarized image 


\section{Skeletonization}

The above binarization process converts fingerprint images into black dots which represent the ridges with a value of 0 , while the white dots signify the valleys with a value of 1 . On the hand, skeletonization (thinning) is a technique that is normally used on binarized images by reducing the thickness of a certain pattern shape until it is represented by 1-pixel wide lines. The method was used in this study, was adopted from the work of (Jun-Sik Kwon, Jun-Woong Gi, \& Eung-Kwan Kang, 2001). This thinning procedure is performed separately on every pixel $\mathrm{P}_{\mathrm{i}}$ appearing as black dots, highlighting the ridges of the fingerprint, by examining the nearest neighbours around it, as shown in Figure 7. In order to make the right decision about the pixel $P_{i}$, three rules were set up to test if the pixel $\mathrm{P}_{\mathrm{i}}$ can be removed from the ridge without affecting the flow and connectivity of the lines while still preserving the ridge endings.

\begin{tabular}{|l|l|l|}
\hline$P_{1}$ & $P_{2}$ & $P_{3}$ \\
\hline$P_{8}$ & $P_{i}$ & $P_{4}$ \\
\hline$P_{7}$ & $P_{6}$ & $P_{5}$ \\
\hline
\end{tabular}

Figure 7. A $3 \times 3$ pixel mask of the nearest neighbours mapped around $P_{i}$

This set of rules can be described as follows:

\section{Rule 1}

$$
\begin{array}{cl}
\text { i. } & 2 \leq D\left(P_{i}\right) \leq 6 . \\
\text { ii. } & T_{r}\left(P_{i}\right)=1 . \\
\text { iii. } & P_{2} \times P_{4} \times P_{6}=0 . \\
\text { iv. } & P_{4} \times P_{6} \times P_{8}=0 .
\end{array}
$$

Where $\mathrm{D}(\mathrm{Pi})$ represents the number of neighbours with values of 0 :

$$
D\left(P_{i}\right)=P 1+P 2+\cdots+P 8
$$

$\mathrm{T}_{\mathrm{r}}\left(\mathrm{P}_{\mathrm{i}}\right)$ is the number of transactions from black to white patterns in the neighbours:

$$
T_{r}\left(P_{i}\right)=(P 1 \mid P 2) \&(\sim P 1)+(P 2 \mid P 3) \&(\sim P 2)+\cdots+(P 8 \mid P 1) \&(\sim P 8)
$$

Examples of rule 1 are shown in Figure 8

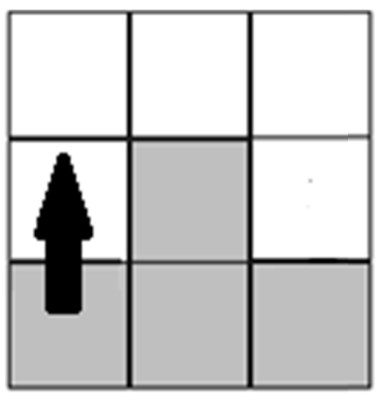

(a)

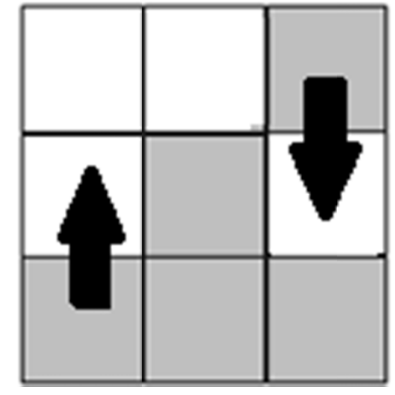

(b)

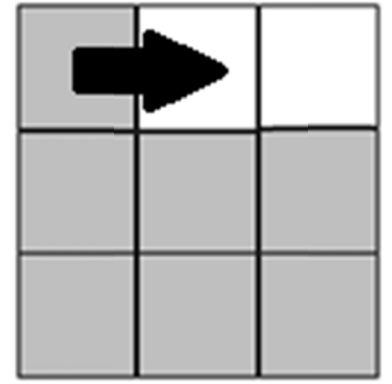

(c)

Figure 8. (a), (b) and (c) Examples of rule 1

In the above example, rule 1 obtained the value for $D\left(P_{i}\right)$ in Figure 8 (a), (b) and (c) is $3,4,6$ respectively, and the value of $\mathrm{T}_{\mathrm{r}}\left(\mathrm{P}_{\mathrm{i}}\right)$ in Figure $8(\mathrm{a}),(\mathrm{b})$ and (c) is $1,2,3$ respectively. Although the above rule reduced the size of both horizontal and vertical lines, it may not truly represent slanting lines that are 2-pixels wide. To solve this problem, another set of rules was used to neutralize that effect. 
Rule 2:

$$
\begin{array}{cl}
\text { i. } & 3 \leq D\left(P_{i} \leq 6 .\right. \\
\text { ii. } & T_{r}=\left(P_{i}\right)=1 . \\
\text { iii. } & P_{2} \times P_{4} \times P_{8}=0 . \\
\text { iv. } & P_{2} \times P_{6} \times P_{8}=0 .
\end{array}
$$

Rule 2 prevents erasing the 2-pixel wide lines, but it cannot produce 1-pixel wide lines. In order to produce 1-pixel wide lines, rule 3 is implemented with following conditions:

Rule 3:

$$
\begin{aligned}
\text { i. } & P_{1} \times P_{8} \times P_{6}=1 \& P_{3}=0 . \\
\text { ii. } & P_{3} \times P_{4} \times P_{6}=1 \& P_{1}=0 . \\
\text { iii. } & P_{5} \times P_{6} \times P_{8}=1 \& P_{3}=0 . \\
\text { iv. } & P_{4} \times P_{6} \times P_{7}=1 \& P_{1}=0 .
\end{aligned}
$$

Patterns in which pixels $P_{i}$ are removed in the second Skeletonization can be seen in Figure 9.

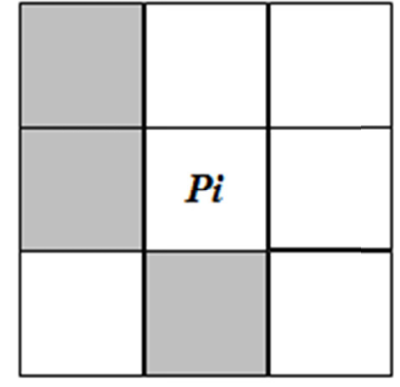

(a)

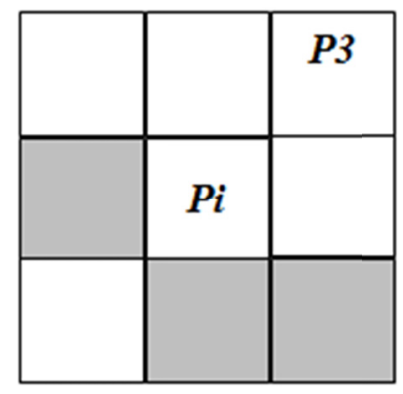

(c)

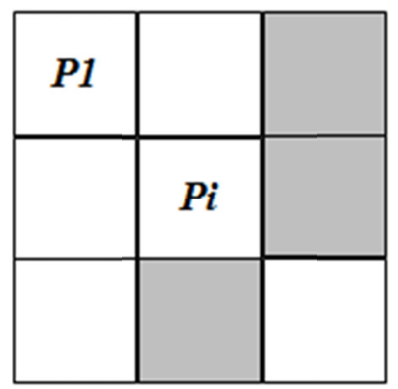

(b)

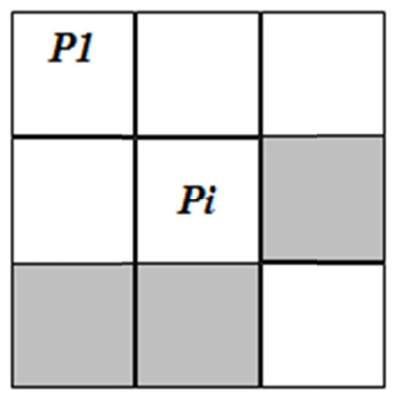

(d)

Figure 10. Skeletonization: (a - d) Examples of rule 3 effects

The examination of the pixels $P_{i}$ is done in repetition where the first two rule sets are applied in turns. Upon completion of each iteration, the affected pixels are removed from the image. This process is repeated until all pixels are exhausted. The second Skeletonization process removes the remaining pixels to produce the 1-pixel wide lines. This process takes only one iteration. The image is then converted back resulting in a skeleton of the binarized fingerprint image. The flow diagram is shown in Figure 10. 


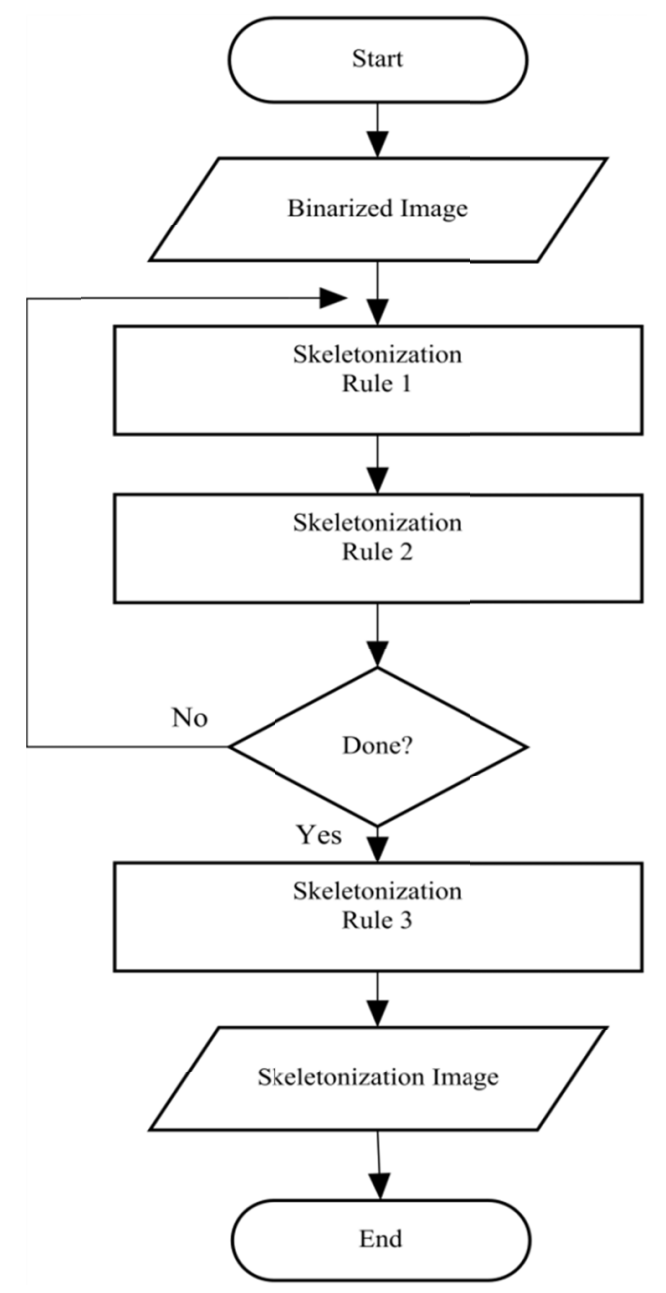

Figure 10. Flow diagram of the Skeletonization process

An example of fingerprint image that has gone through the Skeletonization process is provided in Figure 11.

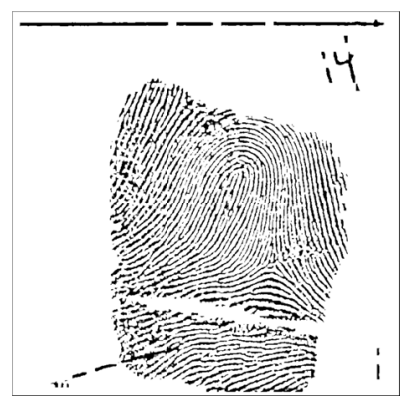

(a)

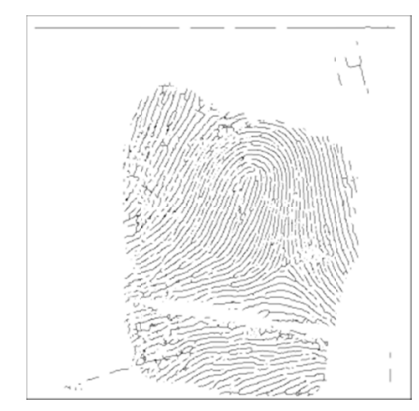

(b)

Figure 11. Skeletonization: (a) The input Binarized image (b) Skeleton image

\section{Proposed Image Fusion}

Discrete Cosine Transform (DCT) is used to fuse between Histogram Equalized image and Skeleton image to produce an enhanced image with a clear ridge structure and an acceptable contrast. Firstly, both input fingerprint images are divided into non-overlapping blocks of $8 \times 8$ pixels. For each block, a two dimensional DCT is calculated using the following equation: 


$$
\operatorname{dct}(i, j)=\alpha(i) \alpha(j) \sum_{x=0}^{N-1} \sum_{y=0}^{N-1} I(x, y) \cos \left[\frac{\pi(2 x+1) i}{2 N}\right] \cos \left[\frac{\pi(2 y+1) j}{2 N}\right]
$$

where

$$
\begin{gathered}
\qquad \alpha(i)= \begin{cases}\sqrt{\frac{1}{N}} & \text { for } i=0 \\
\sqrt{\frac{2}{N}} & \text { for } i \neq 0\end{cases} \\
\alpha(j)= \begin{cases}\sqrt{\frac{1}{N}} & \text { for } j=0 \\
\sqrt{\frac{2}{N}} & \text { for } j \neq 0\end{cases} \\
\text { for } i, j=0,1,2, \ldots, N-1, \text { where } N=8 .
\end{gathered}
$$

Subsequently, the mean and variance for each $\mathrm{N} \times \mathrm{N}$ block are calculated from their DCT coefficients using equations (15) and (16) respectively as derived from (Haghighat, Aghagolzadeh, \& Seyedarabi, 2011):

$$
\begin{aligned}
& \operatorname{mean}_{d c t}=\frac{1}{N^{2}} \sum_{u=0}^{N-1} \sum_{v=0}^{N-1} d c t(u, v) \\
& \operatorname{var}_{d c t}=\frac{1}{N^{2}} \sum_{u=0}^{N-1} \sum_{v=0}^{N-1}\left(d c t(u, v)-\text { Mean }_{d c t}\right)^{2}
\end{aligned}
$$

The fusion process using DCT is based on the maximum information for each pixel block of both DCT images (Histogram Equalization (HE) and Skeletonization (SK)). The high information used in this study depended on the variance values of each block. In other words, the fusion process compared the variance values for each block, where the block with the highest value was included and the other ignored. From both processed DCT images, a final DCT image was obtained using equation (17) below.

$$
d c t_{f u s e d}(i, j)= \begin{cases}d c t_{H E}(i, j) & \text { if } \operatorname{var}_{d c t(H E)}>\operatorname{var}_{d c t(S K)} \\ d c t_{S K}(i, j) & \text { if } \operatorname{var}_{d c t(H E)}<\operatorname{var}_{d c t(S K)}\end{cases}
$$

In order to convert the $\mathrm{dct}_{\text {fused }}$ images that were obtained back to fusion images with clear ridges, an inverse transform was applied and calculated using the formula in (18).

$$
I(x, y)=\sum_{x=0}^{N-1} \sum_{y=0}^{N-1} \alpha(i) \alpha(j) d c t_{f u s e d}(i, j) \cos \left[\frac{\pi(2 x+1) i}{2 N}\right] \cos \left[\frac{\pi(2 y+1) j}{2 N}\right]
$$

$$
\text { For } \mathrm{i}, \mathrm{j}=0,1,2, \ldots, \mathrm{N}-1 \text {. }
$$

The summary of the fusion process is explained in the diagram in Figure 12 below: 


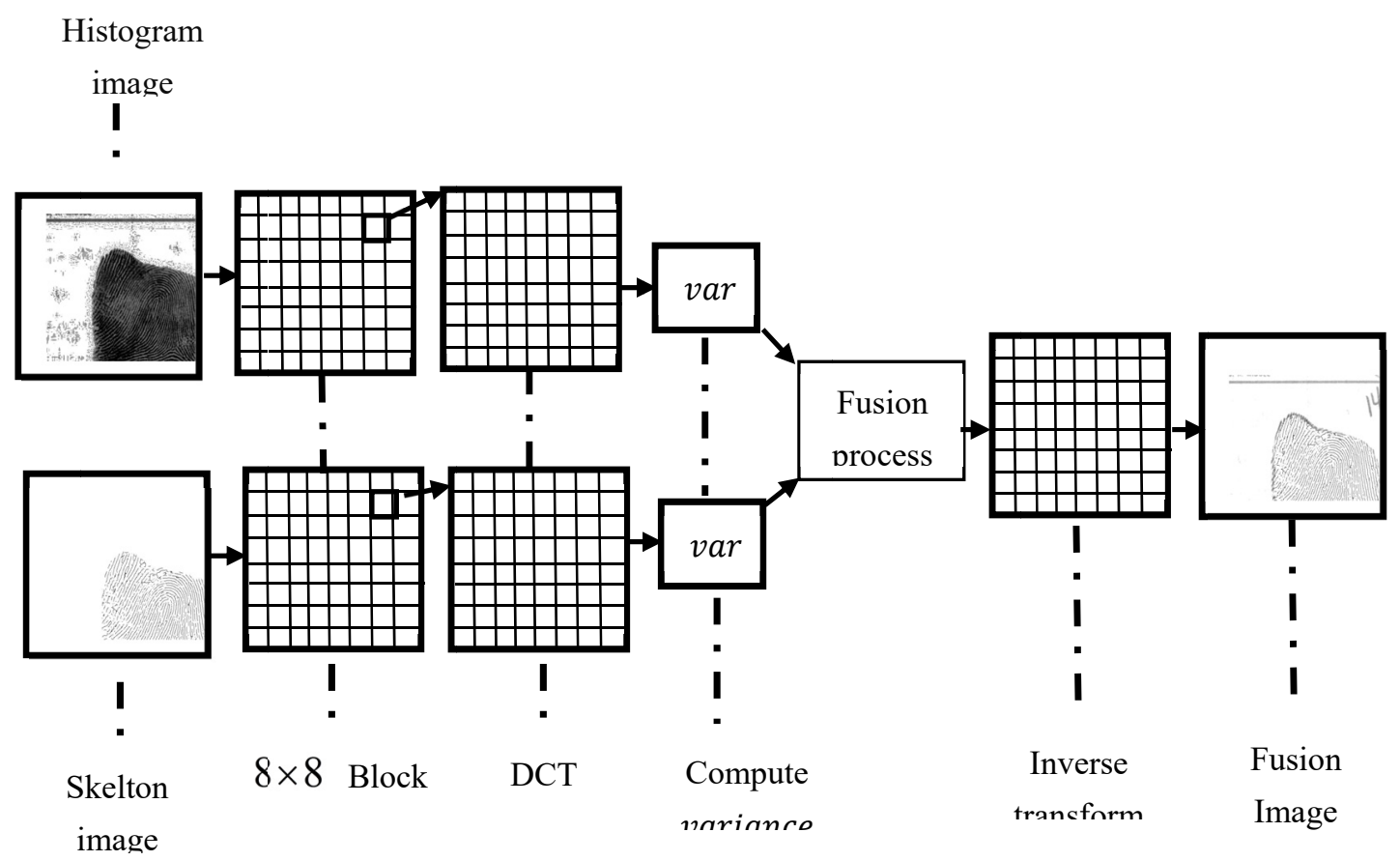

Figure 12. Summary of the DCT fusion process

The output of the fusion process was a enhanced greyscale image with clear ridge flow and high contrast as shown in Figure 13.

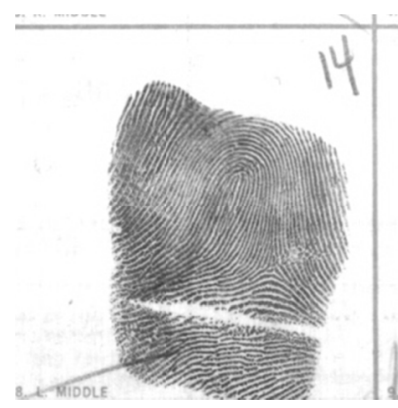

(a)

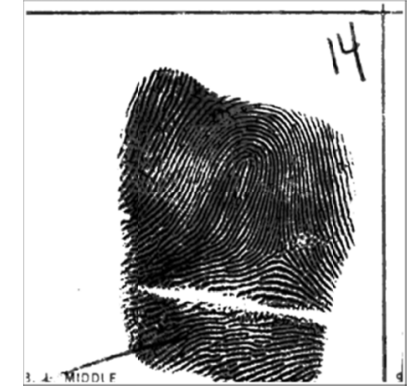

(b)

Figure 13. (a) Original image (b) Fused image (NIST F0000008)

\section{Experimental Results}

Our methodology has been tested using different quality images obtained from National Institute of Standard and Technology (NIST) Special Database 14. Fingerprint images from the NIST Special Database 14 are raw data of various qualities: clear, low-contrast, cut and wet. The evaluation of the current method based on virtual inspection of different quality images. In good quality images, the effect of the Binarization and Skeletonization processes became clearly visible by increasing the contrast between the prints' ridges and valleys. Using the Binarization process a value of 0 was assigned to black pixels, representing the fingerprint ridges, and a value of 1 was assigned to white pixels, representing the valleys. The Skeletonization processes replaced more than one successive wide pixel by just one pixel wide line as shown in Fig ure14. 


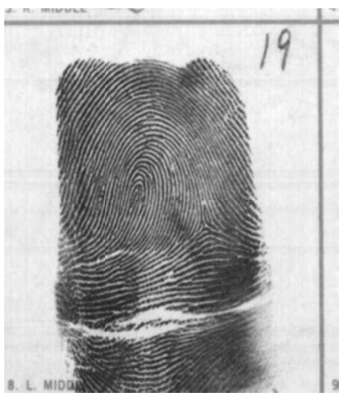

(a)

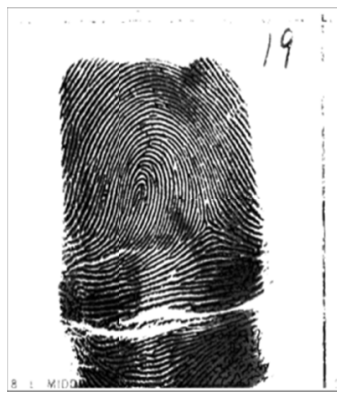

(b)

Figure 14. Results of the current method on good quality image where (a) Original image (b) Enhanced image

(Source NIST Special Database 14 (File name: F0000118)

The image contrast was achieved based on the relationship between the pixels with the brightest and darkest intensity. The images with high intensity of white pixels are referred to as the brightest images, or low contrast images. These images are the result of the illumination effect when using a capture device in live scans, or a weaker impression of the fingertip in ink scans. The fingerprint ridges and valleys in low contrast images cannot be seen clearly, and some parts of the images are missing. The current enhancement method appeared to be effective in improving the quality of low contrast images. The Histogram Equalization process increased the darkest pixel intensities and the fusion process recovered most of the missing parts of the fingerprint patterns illustrated in Figure15 and Figure 16.

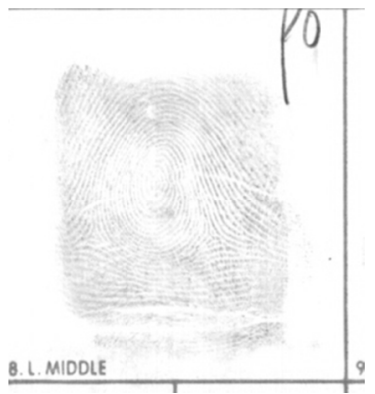

(a)

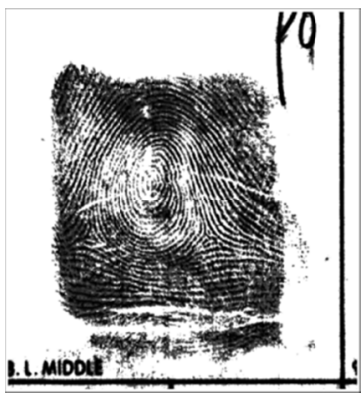

(b)

Figure 15. Results of the current method on low contrast image where (a) Original image (b) Enhanced image (Source NIST Special Database 14 (File name F0000958)

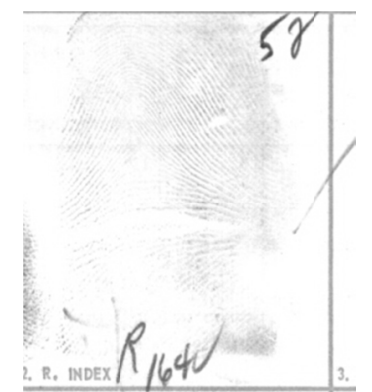

(a)

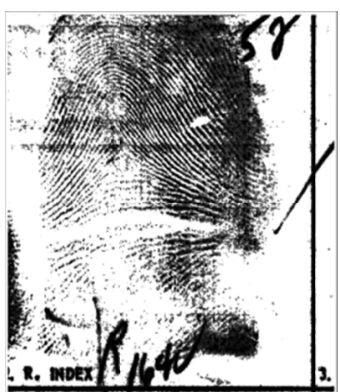

(b)

Figure 16. Results of the current method on low contrast image where (a) Original image (b) Enhanced image (Source NIST Special Database 14 (File name F0001972)

Environmental conditions such as temperature, humidity and pressure also affect the quality of the fingerprint images. Dry skin tends to lead to uneven contact of a finger's ridges with a scanner plate's surface, resulting in 
broken ridges and many white pixels replacing the ridge structure. Conversely, the valleys of a fingertip with oily skin tend to fill up with moisture, making them appear black in an image, similar to a ridge structure. Using the current enhancement method the ridge and valley patterns of prints were smoothed and able to improve the quality of these images as shown in Figure 17 and Figure 18.

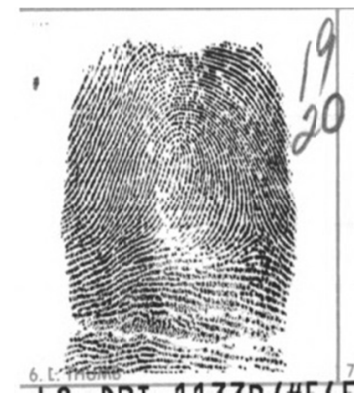

(a)

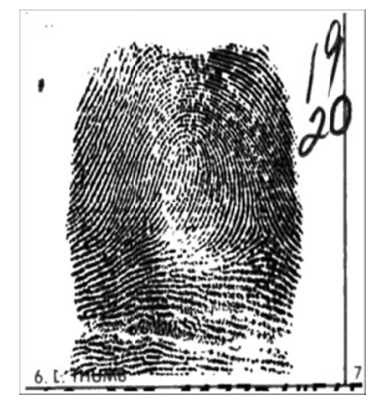

(b)

Figure 17. Results of the current method cut image where (a) Original image (b) Enhanced image (Source NIST Special Database 14 (File name: F0000036)

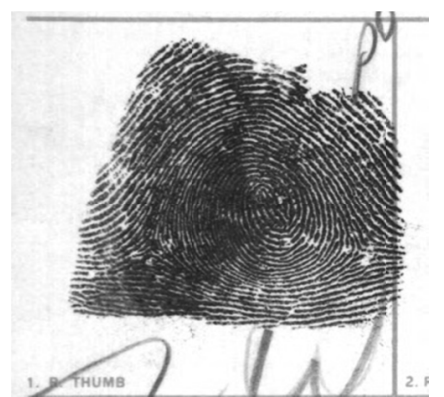

(a)

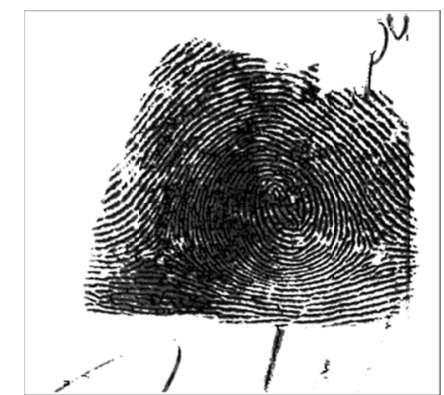

(b)

Figure 18. Results of the current method wet image where (a) Original image (b) Enhanced image (Source NIST Special Database 14 (File name: F0000411)

\section{Conclusion}

We have presented a new combination methodology of fingerprint image enhancement. These shortcomings are overcome and the image quality is extraordinarily improved by applying various enhancement techniques. Histogram Equalization process is used to increase the image contrast. Furthermore, Binarization followed by Skeletonization processes is carried out to eliminate the problem related to disconnectivity in the fingerprint's patterns. By taking the advantage of the previous enhancement processes, fusion technique using DCT is implemented on the results acquired from Histogram Equalization and Skeletonization processes. Consequently, an image with high contrast and connective patterns is achieved.

\section{References}

Çavuşoğlu, A., \& Görgünoğlu, S. (2008). A fast fingerprint image enhancement algorithm using a parabolic mask. Computers \& Electrical Engineering, 34(3), 250-256. http://doi.org/10.1016/j.compeleceng.2006.11.006

Fu, M., Huang, J., \& Xu, J. (2013). A Novel Fingerprint Image Preprocessing Algorithm. In Applied Mechanics and Materials (Vol. 347-350, pp. 2528-2532). Retrieved from http://www.scientific.net/AMM.347-350.2528

Haghighat, M. B. A., Aghagolzadeh, A., \& Seyedarabi, H. (2011). Multi-focus image fusion for visual sensor networks in DCT domain. Computers \& Electrical Engineering, 37(5), 789-797. http://doi.org/10.1016/j.compeleceng.2011.04.016

Hanoon, M. F. (2011). Contrast Fingerprint Enhancement Based on Histogram Equalization Followed By Bit 
Reduction of Vector Quantization, 11(5), 116-123.

Jain, A. (1998). Fingerprint image enhancement: algorithm and performance evaluation. IEEE Transactions on Pattern Analysis and Machine Intelligence, 20(8), 777-789. http://doi.org/10.1109/34.709565

Jain, A. K., Pankanti, S., \& Bolle, R. (1997). An identity-authentication system using fingerprints. Proceedings of the IEEE, 85(9), 1365-1388. http://doi.org/10.1109/5.628674

Jun-Sik Kwon, Jun-Woong Gi, \& Eung-Kwan Kang. (2001). An enhanced thinning algorithm using parallel processing. In Proceedings 2001 International Conference on Image Processing (Cat. No.01CH37205) (Vol. 2, pp. 752-755). IEEE. http://doi.org/10.1109/ICIP.2001.958228

Yager, N., \& Amin, A. (2004a). Fingerprint classification: a review. Pattern Analysis \& Applications, 7(1), 77-93. http://doi.org/10.1007/s10044-004-0204-7

Yager, N., \& Amin, A. (2004b). Fingerprint verification based on minutiae features: a review. Pattern Analysis \& Applications, 7(1), 94-113. http://doi.org/10.1007/s10044-003-0201-2

\section{Copyrights}

Copyright for this article is retained by the authors, with first publication rights granted to the journal. This is an open-access article distributed under the terms and conditions of the Creative Commons Attribution license (http://creativecommons.org/licenses/by/4.0/). 